\title{
ZnS-Based Photonic Crystals
}

\author{
W. Park $\left.{ }^{1}\right)^{*}$ ), J. S. King, C. W. NefF, C. Liddell, and C. J. Summers ${ }^{2}$ ) \\ Phosphor Technology Center of Excellence, School of Materials Science and Engineering, \\ Georgia Institute of Technology, Atlanta, GA 30332-0245, USA
}

(Received July 27, 2001; accepted September 30, 2001)

Subject classification: 71.20.Nr; 78.20.Ci; 85.60.Bt; S8.11

\begin{abstract}
Recent studies of the application of II-VI materials to the fabrication of photonic crystals are reported. Modeling studies show the potential of $\mathrm{ZnS}$ photonic crystals to provide a new method of controlling the emission characteristics of this material system so as to enhance color, intensity and decay time. The same structures are also shown to possess giant refraction and dispersion properties that can be used to control (collect, focus and steer) light. The fabrication of these period structures is addressed. Two approaches are being considered: the fabrication of two-dimensional $\mathrm{ZnS}$ photonic crystals by conventional electron beam lithography and the formation of three-dimensional $\mathrm{ZnS}$ photonic crystals by cost-effective self-assembly methods. ZnS and related II-VI compounds are very attractive for applications in photonic crystal devices operating in the visible and near IR region due to their high indices of refraction and large bandgaps that make them highly transparent in the visible. We report recent studies on the self-assembly of nanoparticles and subsequent $\mathrm{ZnS}$ infiltration techniques that can be used to control the emission and out-coupling properties of phosphors embedded in a photonic crystal.
\end{abstract}

Introduction During the last decade a new class of materials, "photonic crystals" (PCs), has been created by the self-assembly of nanoparticles and the nano-patterning of optical materials $[1,2]$. PCs are materials with a patterned periodic dielectric constant that creates an optical bandgap in the material. As a result, photons with energies lying within this band cannot propagate unless a "defect" causes an allowed propagation state within the bandgap. Thus, point, line, or planar defects can act, respectively, as optical cavities, waveguides, or mirrors and offer new mechanisms for the control of light. Improved control over the generation and emission properties of light can be used to enhance phosphor efficiencies, optical out-coupling, and to develop wavelengthtunable low threshold luminescent materials. The flexibility of PC designs strongly suggests the potential of integrating different materials and material functions to produce a wide variety of new electro-optical systems. Thus, PCs are expected to have a revolutionary impact on optical signal processing and data transmission systems for communications and displays.

By using optically active materials as PC building blocks, it should be possible to achieve greater control over the emission, spectral purity, and dispersion properties of light so as to realize unique electro-optical and optical devices. However, to effectively realize novel photonic materials and devices utilizing PC concepts, it is essential to have the capability to predict the photonic band structure and its effects on the optical properties absorption, dispersion, and luminescence. To guide these investigations we have

1) e-mail: wpark@colorado.edu

*) Present address: Department of Electrical and Computer Engineering, University of Colorado, Boulder, CO 80309-0425, USA.

2) e-mail: chris.summers@mse.gatech.edu 
performed a comprehensive theoretical study of the photonic band structures of different lattice designs in order to select viable PC lattice structures for specific purposes and device properties. Experimentally, we have investigated the properties of PCs formed by self-assembled opals and inverse opals.

Modeling of Photonic Crystal Structures For these studies we have used modifications of the codes available in the literature to calculate the dependence of the photonic band structure on sample properties and structure, i.e. the host refractive index, emission properties and the type of PC structure and its geometry (lattice spacing and hole diameter). From this basis we have performed ab-initio photonic band calculations for $\mathrm{ZnS}: \mathrm{Mn}$ in two- and three-dimensional photonic crystals. As is well known, $\mathrm{ZnS}: \mathrm{Mn}$ is the most efficient electroluminescent phosphor exhibiting a broad emission band centered around $590 \mathrm{~nm}$ due to the intra-ionic transition of $\mathrm{Mn}^{2+}$ ions. By patterning periodic air holes on a $\mathrm{ZnS}$ : Mn thin film, one can create $2 \mathrm{D}$ photonic crystals. When the photonic bandgap is made to overlap the $\mathrm{ZnS}: \mathrm{Mn}$ emission band, the propagation of the Mn luminescence within the film will be forbidden, causing the light trapped within the film to dissipate outside. This phenomenon could be used to obtain highly efficient out-coupling. It would be ideal to have 3D crystals that exhibit a complete photonic bandgap and thus provide full three-dimensional confinement of light. In such cases, it will be possible to design microcavities with very high $Q$-factors which can then be used to dramatically enhance luminescence efficiency. While the 3D crystal structure with full photonic bandgap is still being sought, certain 3D photonic crystals exhibit large pseudo-gaps for specific directions, opening possibilities of photonic band engineering without the full bandgap.

More advanced photonic band engineering can be achieved by introducing a photonic defect state within the bandgap. By changing the geometry of the defect, the defect band position can be changed, enabling the control of emission color and an enhancement in efficiency without changing the phosphor material itself. This provides a new pathway to controlling phosphor chromaticity without changing the phosphor materials or activator ions. Furthermore, the correct type of defect structure can be used to form a microcavity that can enhance the phosphor efficiency dramatically. However, because of the size of these structures they must be fabricated by electron beam lithography and reactive ion etching, which has lead to a strong interest in developing other fabrication techniques.

Numerical simulations The numerical tools used for photonic crystal simulations are based on the plane wave method [3] and the order- $N$ method [4, 5]. The former approach transforms the Maxwell equations into an eigenvalue equation and then uses the plane wave basis set to solve it. This method is especially efficient for obtaining photonic band structure, mode energy, steady-state field profiles, etc. The latter approach involves direct discretization of real space and time and starts with the two time-dependent Maxwell equations. Often referred to as the finite-difference time-domain method, this technique is especially powerful for the calculation of transmission and reflection spectra and also for investigating the temporal behavior of physical quantities. In this approach, it is very important to eliminate any unphysical reflections from the artificial boundaries set by the simulation. This is achieved by applying the perfectly matched layer (PML) boundary condition at all boundaries of the simulation. Inside 
the perfectly matched layers, the $E$ and $H$ fields are attenuated and eventually absorbed so as to prevent any reflections.

Light emission properties The formation of microcavities in 2D and 3D PC structures gives control over the emission energy, linewidth and spontaneous emission rate, such that these properties become as much a property of the cavity design as the material itself. This allows more highly saturated colors, faster response times, and lower threshold devices. We have therefore studied the control that can be obtained in the emission and out-coupling properties of a PC. By performing ab-initio photonic band calculations for luminescent thin films patterned with a two-dimensional crystal structure, such as a periodic array of air holes, one can create photonic bandgaps whose positions can be adjusted by changing the periodicity, so that the photonic bandgap overlaps the emission band. The propagation of the luminescence within the film will then be forbidden, causing the light trapped within the film to dissipate outside. An investigation of this phenomenon can potentially result in more efficient out-coupling of light from a thin film. More advanced photonic band engineering can be achieved by introducing a photonic defect state within the bandgap to create an optical cavity. By changing the geometry of the defect, the cavity properties can be changed, enabling control over the emission color and an enhancement in efficiency without changing the phosphor material itself.

Our ab-initio photonic band calculations for several simple two-dimensional lattices in $\mathrm{ZnS}$ : Mn have shown that a square lattice is not suitable for PC devices, but a triangular lattice exhibits a wide photonic bandgap between the first and second bands for TE polarization, while no gap was formed for TM polarization, as shown in Fig. 1. By adjusting the lattice parameter, it was found that the TE bandgap completely overlapped the Mn emission band from 565 to $690 \mathrm{~nm}$ when $a=200 \mathrm{~nm}$ and $r=60 \mathrm{~nm}$. This suggests that it will be possible to obtain highly efficient out-coupling by incorporating a triangular lattice of air holes in $\mathrm{ZnS}: \mathrm{Mn}$. We have also calculated the transmission characteristics and mode properties of these structures by using the order- $N$ method. Figure 2 shows the transmission spectrum calculated for light incident in the $\Gamma-X$ direction. The partial band structure in the $\Gamma-\mathrm{X}$ direction is also displayed for compar-
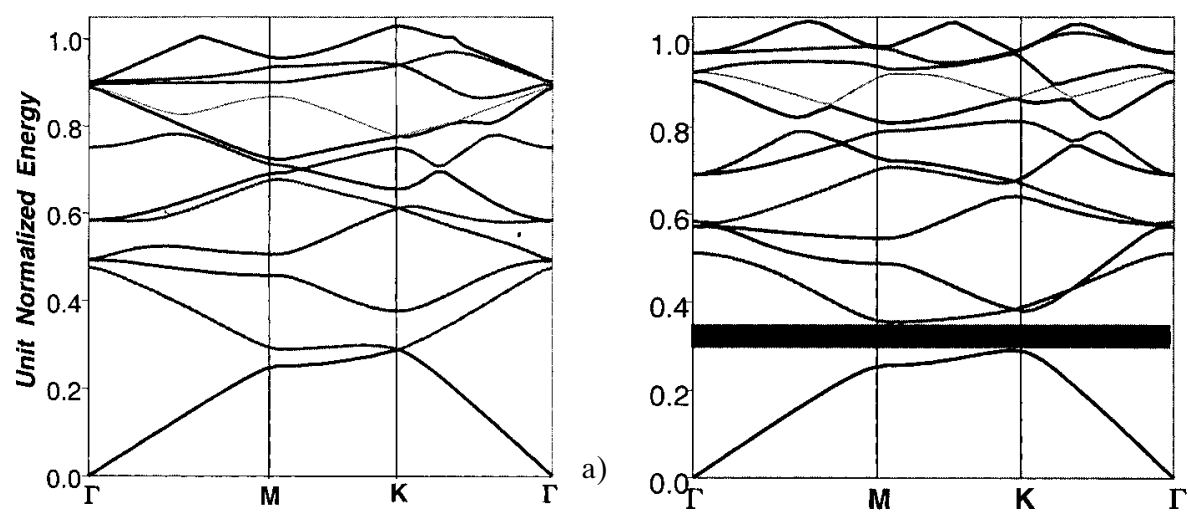

b)

Fig. 1. Photonic band structure of a two-dimensional triangular lattice of air holes in $\mathrm{ZnS}$ : Mn for a) TM and b) TE polarization 

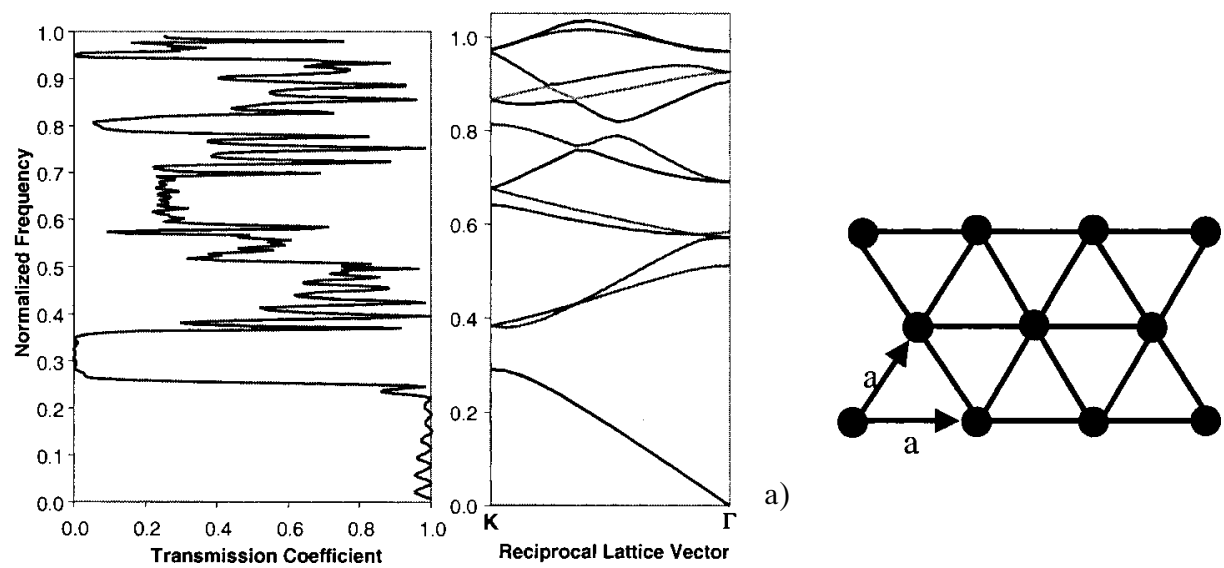

a)

Fig. 2. a) Calculated transmission spectrum for a $2 \mathrm{D}$ triangular lattice. The light is assumed to be incident in the $\mathrm{K}-\Gamma$ direction and the corresponding band diagram is also shown. b) Schematic of triangular lattice of air holes

ison. The calculated spectrum shows complete suppression of transmission in the region precisely coincident with the gap in the band structure, clearly demonstrating the effect of photonic bands on the luminescent properties of $\mathrm{ZnS}: \mathrm{Mn}$.

We next performed a defect band calculation for a triangular lattice of air holes patterned in $\mathrm{ZnS}: \mathrm{Mn}$ which contains a missing air hole. Ab-initio supercell calculations predict that a defect band will be formed when one air hole is missing and show that the defect mode can be tuned by changing the defect geometry. As $r$ is increased from 0 to $100 \mathrm{~nm}$, the energy of the defect band increases from a corresponding wavelength of 608 to $552 \mathrm{~nm}$. As shown in Figs. 3a and b, a sharp line emission can be extracted
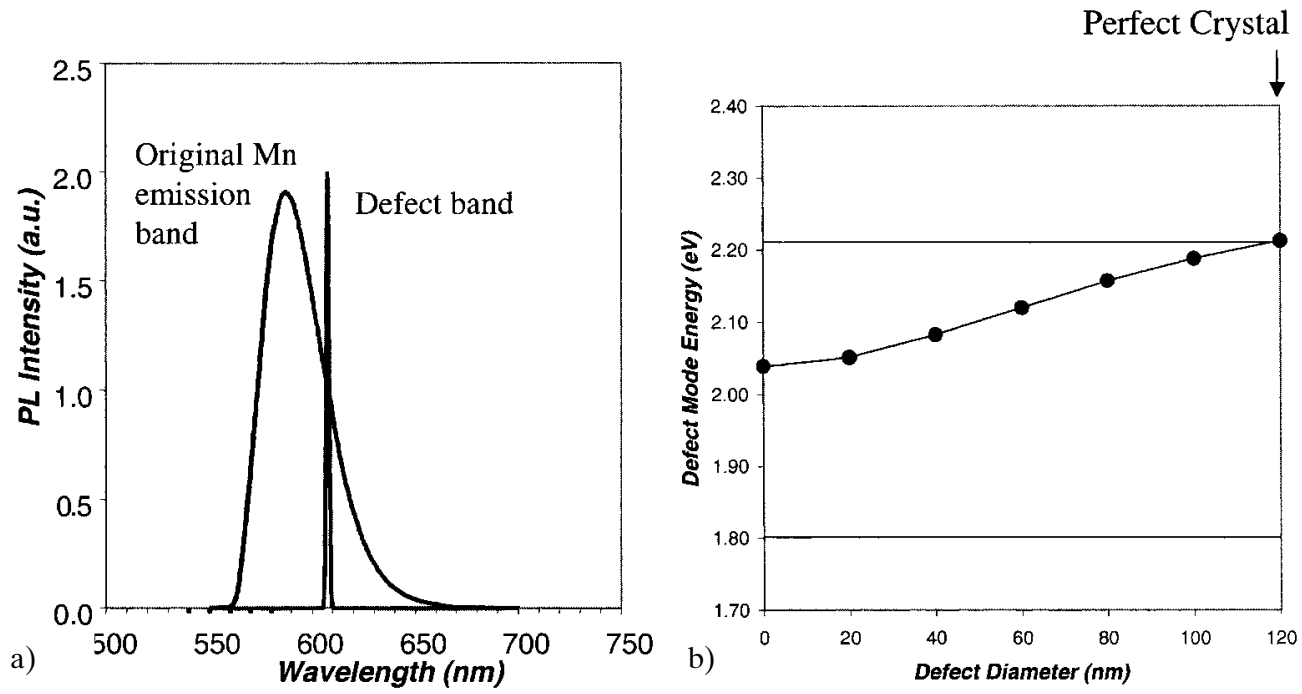

Fig. 3. a) Emission spectrum of $\mathrm{ZnS}: \mathrm{Mn}$ and the defect mode of $\mathrm{ZnS} /$ air PC introduced by a missing air hole. b) Energy of the defect band as a function of the defect radius 
from the broad $\mathrm{ZnS}$ :Mn emission by introducing a missing air hole and can be tuned over a significant range of wavelengths. Thus this phenomenon opens a new way to control emission color chromaticity without changing the phosphor materials or activator ions. Furthermore, enhanced spontaneous emission probability is expected due to the Purcell effect [6] since the dimension of the cavity is on the order of the emission wavelength.

Refractive properties Additionally, it is demonstrated in this study that these structures also exhibit a wide range of other properties that can be used to control the reflective, refractive and diffractive properties of light, as well as to switch and modulate light. Very high dispersion (superprism) properties were first reported by Kosaka et al. [7, 8] for a complicated three-dimensional structure based in a $\mathrm{Si} / \mathrm{SiO}_{2}$ lattice that was fabricated by the sequential deposition of $\mathrm{Si}$ and $\mathrm{SiO}_{2}$ on silicon patterned substrates. We show in this study that in fact the same results can be achieved in far simpler structures, for example consisting of a periodic lattice of air holes formed in both high and low index films, such as $\mathrm{Si}$ and $\mathrm{ZnS}$. In the paper, we demonstrate these effects for a triangular lattice, however, similar effects are also predicted for a wide variety of lattices (square, rectangular, hexagonal). Furthermore, it is demonstrated that with these structures a wide variety of giant refraction and diffraction properties can be achieved to collect, focus, disperse, switch and steer light.

The observation of these properties demonstrates the versatility of these concepts for initiating many new types of opto-electronic circuits and optical materials. It should also be noted that $2 \mathrm{D}$ structures have many advantages compared to $3 \mathrm{D}$ structures. Their fabrication process is more straightforward, the number of allowed modes is lower due to the simpler band structures, and there is no mixing between TE and TM polarizations.

One of the most interesting and technologically important properties of photonic crystals is their ability to exhibit highly non-linear dispersion at wavelengths close to the photonic bandgap. This was first demonstrated by Lin et al. [9], who observed a significant change in the dispersion characteristics in photonic crystals designed for radio wave propagation. Recently, Kosaka et al. [7, 8] have further pursued this work and demonstrated near infrared photonic crystals that show extremely non-linear dispersion characteristics. Light beam steering that reached an angle of $50^{\circ}$ was reported for only a $1 \%$ shift in the incident wavelength, that is from 1.0 to $0.99 \mu \mathrm{m}$. This angular dispersion is two orders of magnitude greater than what can be achieved with conventional gratings, and approximately three orders of magnitude greater than what is possible with prisms.

Giant refraction in two-dimensional photonic crystals While Kosaka et al.'s structure was found to exhibit extraordinarily large wavelength and angle dispersion, there exist some drawbacks related to the crystal structure, which was grown by a complicated self-cloning biased sputtering technique. Additionally, all of the demonstrations of superprism phenomena were observed in a two-dimensional cross-section even though this was a three-dimensional structure. Furthermore, all of the experiments were performed for light with either TE or TM polarization, which are not completely separated in 3D structures and thus can suffer from polarization mixing. Another deficiency arises from the fact that a 3D PC invariably has a more complicated band structure, and thus 
has a greater chance of mode branching. Therefore, we propose 2D photonic crystal structures based on $\mathrm{Si}$ and $\mathrm{ZnS}$ that exhibit giant refraction and dispersion. These 2D structures have many advantages compared to their 3D counterparts. The fabrication process is much more straightforward, the number of allowed modes are generally smaller due to the simpler band structures, and there is no mixing between TE and TM polarizations. Here, we present recent results of our simulations, which clearly show the phenomena expected in these structures and which have led to the invention of new electro-optic and display device concepts.

An example of these calculations is shown below. Figure 4a shows the band structure of a ZnS/air 2D PC that exhibits a small photonic bandgap for TE polarization. Strong anisotropic dispersion, the essential characteristic needed for superprism effects, is observed in the third photonic band. Figure $4 \mathrm{~b}$ shows the dispersion diagram calculated for this point. The large anisotropy (outer curve) is clearly seen from its star-like shape. (A similar curve was also obtained for TM polarization.) The propagation direction of light inside the photonic crystal is then obtained from the normal to the dispersion curve, schematically represented in Fig. 4 b.

Using this process, Kosaka et al.'s structure was modeled and our simulations were found to be in good agreement with the experimental data for the $\mathrm{Si} / \mathrm{SiO}_{2}$ structure. Simulations were then performed for the $\mathrm{ZnS} /$ air photonic crystal structure shown in Fig. 5a and the propagation angles for this structure were calculated for various incident angles measured with respect to the $\Gamma-\mathrm{M}$ direction. Figure 5a shows the real space diagram corresponding to this structure and the $\Gamma-\mathrm{K}$ and $\Gamma-\mathrm{M}$ directions. The results of this calculation for light directed principally along the $\Gamma-\mathrm{M}$ direction are shown in Fig. $5 \mathrm{~b}$ where the diamonds and squares represent the TM and TE modes, respectively. The data for the TM polarization was obtained by choosing a similar band point near a normalized energy of 0.43 , for example in the photonic band structure for the TM polarization. The figure clearly demonstrates the giant refraction effect, predicting a drastic change in propagation angle from $0^{\circ}$ to almost $70^{\circ}$ for a small change in incident angle from $0^{\circ}$ to $5^{\circ}$ off the $\Gamma-\mathrm{M}$ direction. We have also demonstrated these effects in a square lattice and proven our model by successfully fitting Kosaka et al.'s data. Furthermore, the dispersion curves are found to be also strongly wavelength dependent, leading to large dispersion (super-

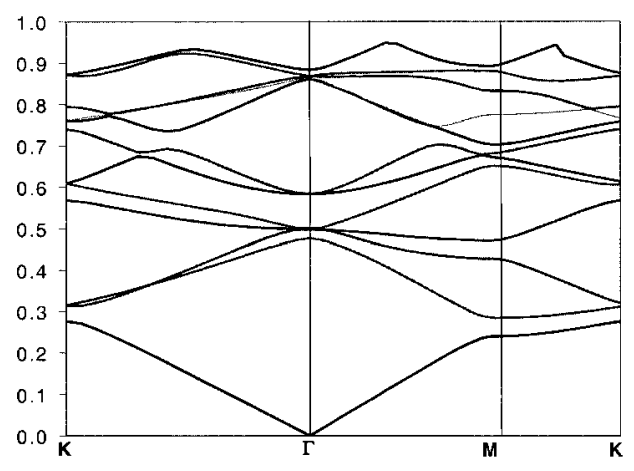

a)

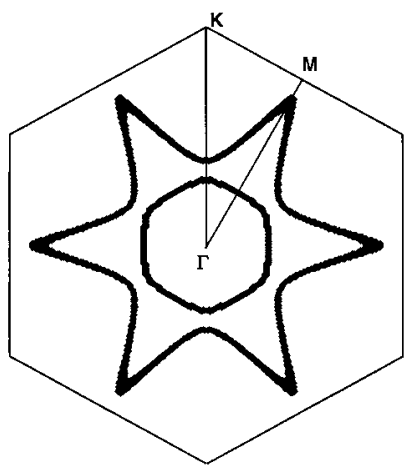

b)

Fig. 4. a) TE photonic bands for a triangular lattice of air holes in ZnS. b) Dispersion diagram at normalized energy 0.43 . (Air hole diameter is $40 \%$ of the lattice constant) 

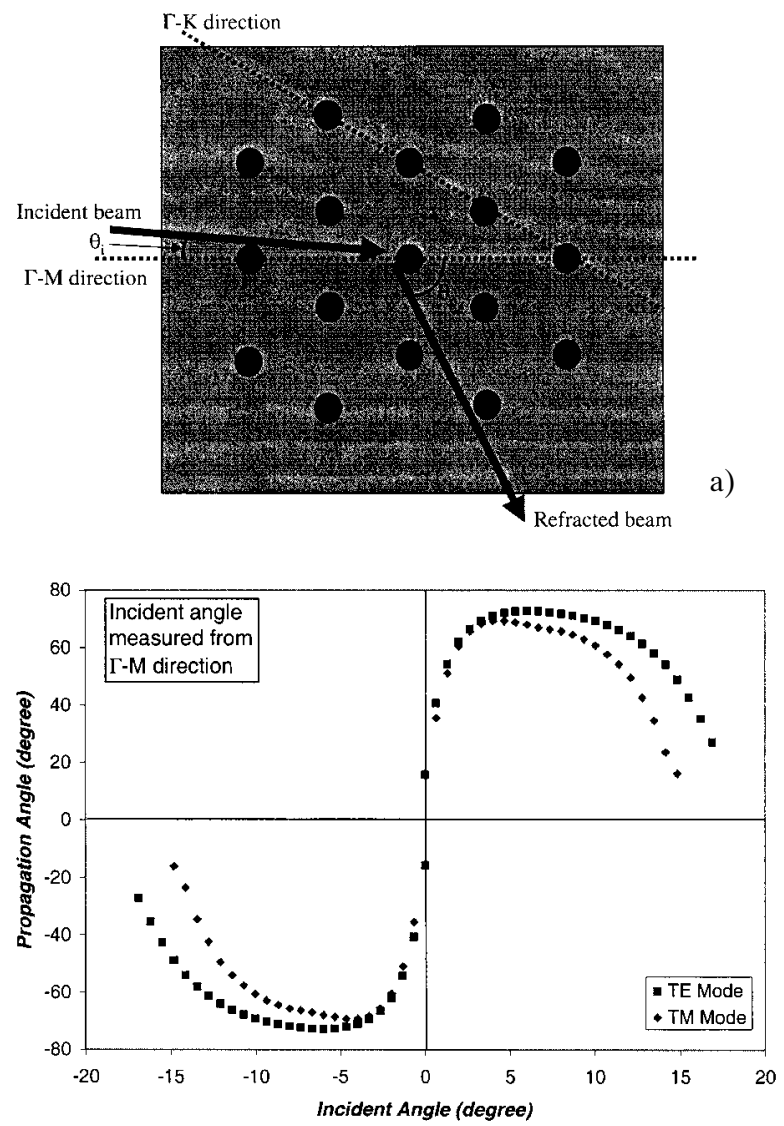

b)

Fig. 5. a) Real space diagram of $2 \mathrm{D}$ triangular lattice of air holes in $\mathrm{ZnS}$. $\Gamma-\mathrm{K}$ and $\Gamma-\mathrm{M}$ directions as well as incident and propagation beam angles are presented. b) Refraction angles calculated for various incident angles measured with respect to the $\Gamma-\mathrm{M}$ direction in a $\mathrm{ZnS} /$ air photonic crystal with $r=0.20 a$

prism) effects. Figures $6 a$ and $b$ demonstrate the large spectral dispersion properties that can be achieved in such a crystal by designing the structure to work at a particular range of wavelengths corresponding to asymmetries in the photonic bandgap structure. It is observed that for light incident with a small angle from the $\Gamma-\mathrm{M}$ direction, a $2 \%$ change in light energy (which corresponds to approximately 5-10 $\mathrm{nm}$ in the visible range) results in a change in refraction angle of almost $30^{\circ}$. It is obvious that this phenomenon will have a wide range of potential applications in various photonic devices.

Experimental Studies Although PC designs have been performed for both $2 \mathrm{D}$ and 3D lattices, most of our experimental studies have been directed towards the fabrication of $3 \mathrm{D}$ structures by self-assembly. Similar to the 2D lattices these structures can also be designed to both suppress luminance and to fabricate microcavity structures. 

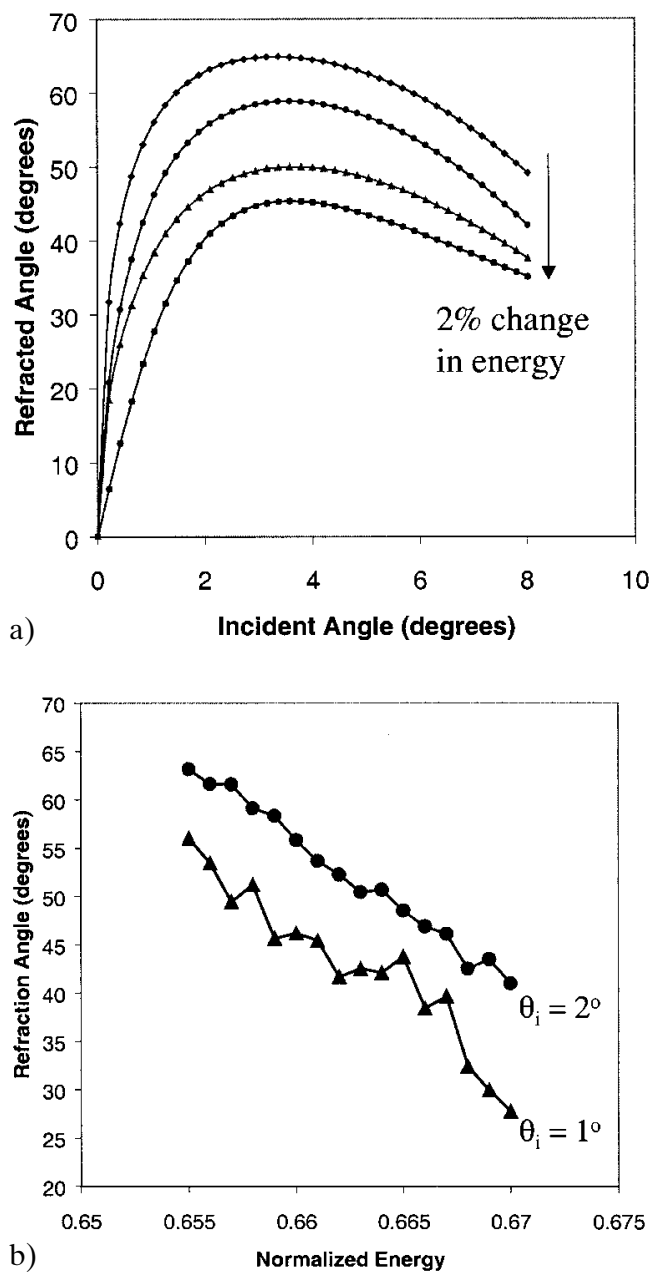

Fig. 6. a) Refraction angle vs. incident angle calculated for various wavelengths. b) Refraction angle as a function of light wavelength which demonstrates the superprism effect expected for a $2 \mathrm{D} \mathrm{ZnS} /$ air photonic crystal structure

Self-assembly techniques Because of the potential of self-assembly techniques to form $3 \mathrm{D}$ structures in a cost-effective manner a number of template methods are being developed to fabricate large area opals and inverse opals [8]. Inverse opals have the advantage of greatly enhancing the refractive index contrast in a photonic crystal because their air-tomaterial volume ratio is much larger than in a simple opal structure. However, the fabrication of inverse opals introduces an entirely new range of strategies into the growth of II-VI materials for these applications. The opal must first be formed, infiltrated with the II-VI material, and then the original opal etched away to leave the inverted II-VI opal, or photonic crystal structure.

Many methods have recently been reported in the literature to fabricate opals. To fabricate an inverse opal structure in $\mathrm{ZnS}: \mathrm{Mn}$ and related phosphors,

such as $\mathrm{ZnS}: \mathrm{Cu}$ etc., we have first made a conventional opal using mono-dispersed silica spheres. The silica nanoparticles were synthesized by the Stober method [10]. As shown in the inset of Fig. 7a, TEM images confirmed highly mono-dispersed size distributions with less than 5\% deviations, for particle sizes ranging from 200 to $1000 \mathrm{~nm}$. The nanoparticles were then self-assembled into an ordered structure by using a specially designed confinement cell made by a lithographic process. To provide a platform for subsequent anneals a silica base plate is used, and to successfully remove the opal from the cell a non-stick surface technique has been developed to coat the inside surface of the top glass plate. Both of these steps significantly reduced the fracturing of the opal when dismounting it from the forming cell. The structure was then sintered at $950{ }^{\circ} \mathrm{C}$ for $2 \mathrm{~h}$ in order to bond the structure together so that it has the structural integrity to withstand further processing. This technique was found to produce larger domain sizes, fewer defects, and higher packing density, and to shorten synthesis time. SEM analysis directly confirmed the formation of synthetic opals with fcc structure. Figure 7a shows an SEM micrograph of an opal. Optical characterizations were then 




a)



Fig. 7. a) SEM micrograph of an opal fabricated by a forced sedimentation technique. Inset: TEM micrograph of monodispersed silica spheres. b) Transmission spectrum for an opal made of $500 \mathrm{~nm}$ silica spheres

made of the opal structures as they offer the best technique to confirm the perfection of the structure. The opals showed well defined stop bands in the transmission spectra due to the Bragg reflections by (111) planes, as shown in Fig. 7b. The position of the stop bands was observed to vary from 510 to $1050 \mathrm{~nm}$ as the size of the silica nanoparticles was increased from 200 to $500 \mathrm{~nm}$.

To achieve a high filling factor for the infiltration of the opal we are currently investigating a number of chemical and photochemical bath deposition methods. In this technique it is necessary to precipitate $\mathrm{ZnS}$ within the dimensions of the opal in order to completely fill the structures. Recently Blanco et al. [11] and Zhou et al. [12] have reported on the inhibition of photo-

luminescence from $\mathrm{CdS}$ and $\mathrm{ZnS}: \mathrm{Mn}$, respectively, by a photonic crystal structure. In these studies Blanco et al. reported a CBD technique in which infiltration was achieved by successive and sequential immersions in aqueous solutions of $\mathrm{CdSO}_{4}$ and $\mathrm{S}=\mathrm{C}\left(\mathrm{NH}_{2}\right)_{4}$ held at $60^{\circ} \mathrm{C}$, followed by an anneal at $700{ }^{\circ} \mathrm{C}$. This technique controls the amount of $\mathrm{CdS}$ inside the opal and prevents formation on the external surface, which could block complete infiltration. A similar technique was also reported by Zhou et al. for $\mathrm{ZnS}: \mathrm{Mn}$. Using the chemical bath technique we have investigated a method to homogeneously precipitate undoped $\mathrm{ZnS}$ and $\mathrm{Cu}$ or $\mathrm{Mn}$-doped $\mathrm{ZnS}$. In this technique a seed solution of nanosized $\mathrm{ZnS}$ particles is prepared and then used to precipitate larger particles. For undoped $\mathrm{ZnS}$ the "seeds" were produced by aging a solution of zinc salt and thioacetamide (TAA). A seeding time of $4 \mathrm{~h}$ was found to produce isolated monodispersed spheres while a time longer than $6 \mathrm{~h}$ produced particle clusters. To control the decomposition of TAA into $\mathrm{H}_{2} \mathrm{~S}$ (aq), the solutions were acidified by the addition of nitric acid, which resulted in a slow release of sulfide ions at room temperature. This reaction can also be accelerated by heating. For doped $\mathrm{ZnS}$, the different hydrolytic 


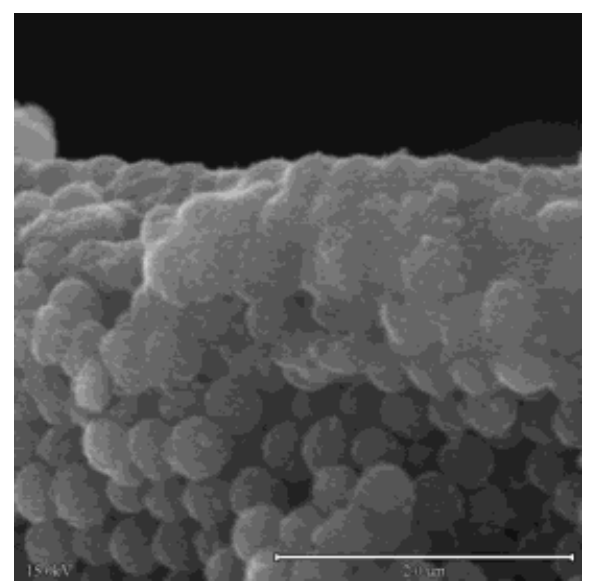

Fig. 8. A cross-sectional SEM micrograph of ZnSinfiltrated opal showing $\mathrm{ZnS}$ filled inside the opal down to approximately 5 layers

stabilities of the two metals in ternary materials make co-precipitation challenging. In our case, the problem is framed in terms of the solubility differences between $\mathrm{ZnS}$, CuS, and MnS. Species having lower solubility in water precipitate more easily. $\mathrm{CuS}$ is less soluble than $\mathrm{ZnS}$, while $\mathrm{MnS}$ is more soluble than $\mathrm{ZnS}$. Doped $\mathrm{ZnS}$ has been produced by adjusting the ratio of metal ions in solution based on the ease of precipitation. SEM characterizations show that $400 \mathrm{~nm}$ to $2 \mu \mathrm{m}$ spherical particles of narrow size distribution as well as particle clusters, networks or gels have been obtained. Though spherical in shape, the particles were to a large extent crystalline, sphalerite-cubic phase, as determined by X-ray diffraction. We believe that this synthesis technique provides a promising route to achieve good infiltration of synthetic opal systems because the $\mathrm{ZnS}$ actually forms when the solution temperature is raised. By choosing appropriate aging conditions nanometer sized seeds are formed in the solution at room temperature, and this homogeneous solution can then be completely infiltrated into the void space of the opal. Then by elevating the solution temperature to $85{ }^{\circ} \mathrm{C}$, the growth of the seeds is activated and the precipitation of $\mathrm{ZnS}$ initiated throughout the opal.

We have also investigated a photochemical deposition technique, which has been pioneered by Ichimura et al., for solar cell applications [13]. Consequently, CdS, a naturally n-type semiconductor, has been extensively studied and only limited results have been published for $\mathrm{ZnS}$ films. We believe that the advantage of this technique for the infiltration of a photonic crystal is that the opal can be fully infiltrated by the solution, and then solidified by illumination. Ideally, growth should occur in a directional manner, thus completely filling the opal. Therefore, we are currently optimizing the photochemical deposition of $\mathrm{ZnS}$ for this application, and investigating the incorporation of dopant ions such as $\mathrm{Cu}$ or $\mathrm{Mn}$ into the host lattice so as to obtain luminescent material. Figure 8 shows an SEM micrograph of an opal infiltrated with ZnS using this deposition technique. In this particular sample, $\mathrm{ZnS}$ filled the voids inside the opal up to approximately 4-5 layers, which we believe to be controllable by adjusting the deposition conditions.

Future Applications There are many applications for PC structures in the II-VI materials system. For example, because the defect structure forms a microcavity, the spontaneous emission characteristics can be significantly changed. As the term "spontaneous" implies, the radiative recombination process occurs with no external means to influence the process and has long been believed to be uncontrollable. However, in an optical cavity with dimensions on the order of the emission wavelength, the optical mode density is significantly altered, which in turn affects the spontaneous emission rate. Purcell 
proposed the first microcavity in the radio frequency regime [6] and current-injection microcavity LEDs based on semiconductor heterostructures [14] and organic materials [15] have been demonstrated in the 1990s. These devices exhibited 2-4 times higher gain, yielding a lower current threshold. The most crucial factor in these devices is the confinement of light. These microcavity devices use Bragg reflectors in one direction but rely on total internal reflection for other directions. Thus, the light is only loosely confined in some directions, limiting the possible enhancement in $Q$-factors. Near perfect light confinement is possible when a microcavity is formed within a photonic crystal structure. The defect structure discussed in previous sections provides a microcavity where the light is tightly confined in the two-dimensional plane and can possess a very high $Q$-factor on the order of several hundred. Thus the spontaneous emission rate can be enhanced dramatically, resulting in an extremely efficient luminescent device. Such a high- $Q$ cavity is also expected to have very low threshold characteristics, making it possible to develop highly sensitive devices, such as detectors and frequency up-converters. Additionally, the extension of these studies to $3 \mathrm{D}$ photonic crystals leads to the exciting prospect of forming three-dimensional high- $Q$ cavities that can further suppress and/or enhance light emission processes. Hirayama et al. [16] have proposed that these effects combined with a directed angular distribution of light emission could lead to athresholdlessa surface emitting laser or LED emission.

Because thin film displays suffer from light trapping due to total internal reflection, their external efficiency is low. Thus, investigations of out-coupling schemes are very relevant to maximizing the output from inorganic and organic electroluminescent displays. The light extraction efficiency from optically pumped light-emitting diode material structures has recently been improved by Boroditsky et al. [17] who fabricated a 2D PC around the active region of an LED. This structure coherently scattered the internally trapped LED emission to yield a sixfold improvement in external efficiency, corresponding to $>70 \%$ external efficiency. One can also envision thin film photonic crystal faceplates designed to enhance forward out-coupling characteristics. The application of these advances to displays will have an important impact in developing sunlight readable systems and also in designing directed light displays.

Conclusions We presented the results of our theoretical and experimental investigations on $2 \mathrm{D}$ and $3 \mathrm{D}$ photonic crystals based on $\mathrm{ZnS}$. These structures were found to exhibit photonic bandgaps in which propagation and generation of light is prohibited. One can then introduce an allowed mode within the bandgap by deliberately introducing a photonic defect. The energy of the defect mode was found to be tunable by changing the size and geometry of the defect, providing a new pathway to control emission color. Furthermore, such defects have dimensions on the order of the optical wavelengths and thus can serve as microcavities which can lead to enhanced luminescence efficiency due to the Purcell effect. The ZnS-based photonic crystals were also found to exhibit strongly non-linear dispersion characteristics, which result in giant refraction and superprism effects. We also investigated the fabrication of $3 \mathrm{D}$ photonic crystals by a self-assembly of nanoparticles technique. We were able to synthesize highly monodispersed nanoparticles and obtain high quality synthetic opals by a forced sedimentation technique. Both SEM and optical characterizations confirmed the formation of highly ordered structures. The opals were then infiltrated with $\mathrm{ZnS}$ by both chemical bath deposition and photochemical deposition techniques. We believe that photonic crystals 
have high potential in realizing a completely new class of photonic devices and that $\mathrm{ZnS}$ and related II-VI compounds are very attractive for applications in photonic crystal devices operating in the visible and near IR region. They have relatively high indices of refraction and can accommodate a variety of activators to exhibit efficient luminescence in various colors. Furthermore, they have high bandgaps and thus are highly transparent in the visible range, which is very important for any application in this spectral region.

Acknowledgements This work was supported by the Phosphor Technology Center of Excellence under DARPA grant MDA972-93-1-0030. J. S. King and C. W. Neff thank the support of Sandia National Laboratories. C. M. Liddell thanks the support of U.S. Department of Defense, Office of Naval Research.

\section{References}

[1] S. Foresi, P. R. Villeneuve, J. Ferrera, E. R. Thoen, G. Steinmeyer, S. Fan, J. D. JoannopouLOUS, L. C. Klimerling, H. I. Smith, and E. P. IPPEn, Nature 390, 143 (1997).

[2] J. WiJnhoven and W. L. Vos, Science 281, 802 (1998).

[3] S. G. Johnson and J. D. Joannopoulos, Opt. Express 8, 173 (2001).

[4] C. T. Chan, Q. L. Yu, and K. M. Ho, Phys. Rev. B 51, 16635 (1995).

[5] A. J. Ward and J. B. Pendry, Comp. Phys. Commun. 128, 590 (2000).

[6] E. M. Purcell, Phys. Rev. 69, 681 (1946).

[7] H. Kosaka, T. Kawashima, A. Tomina, M. Notomi, T. Tamamura, T. Sato, and S. Kawakami, Phys. Rev. B 58, 10096 (1998).

[8] H. Kosaka, T. Kawashima, A. Tomina, M. Notomi, T. Tamamura, T. Sato, and S. Kawakami, Appl. Phys. Lett. 74, 1370 (1999).

[9] S. Y. Lin, V. M. Hietala, L. Wang, and E. D. Jones, Opt. Lett. 21, 1771 (1996).

[10] W. Stober, A. Fink, and E. Bohn, J. Colloid Interface Sci. 26, 62 (1968).

[11] A. Blanco, H. Miguez, F. Meseguer, C. Lopez, F. Lopez-Tejeira, and J. Sanchez-Dehesa, Appl. Phys. Lett. 78, 3181 (2001).

[12] J. Zhou, Y. Zhou, S. Buddhudu, S. L. Ng, Y. L. Lam, and C. H. Kam, Appl. Phys. Lett. 76, 3513 (2000).

[13] M. Ichimura, F. Goto, Y. Ono, and E. Arai, J. Cryst. Growth 198/199, 308 (1999).

[14] E. F. Schubert, Y. H. Yang, A. Y. Cho, L. W. Tu, and G. J. Zygzik, Appl. Phys. Lett. 60, 921 (1992).

[15] T. Nakayama, Y. Itoh, and A. Kakuta, Appl. Phys. Lett. 63, 594 (1993).

[16] H. Hirayama, T. Hamano, and Y. Aoyagi, Appl. Phys. Lett. 69, 791 (1996).

[17] M. Boroditsky, T. F. Krauss, R. Coccioli, R. Vrijen, R. Bhat, and E. Yablonovitch, Appl. Phys. Lett. 75, 1036 (1999). 